\title{
Chemotaxis of cargo-carrying self-propelled particles
}

\author{
Hidde D. Vuijk, Holger Merlitz, Michael Lang, Abhinav Sharma, Jens-Uwe Sommer
}

\begin{abstract}
Active particles with their characteristic feature of self-propulsion are regarded as the simplest models for motility in living systems. The accumulation of active particles in low activity regions has led to the general belief that chemotaxis requires additional features and at least a minimal ability to process information and to control motion. We show that self-propelled particles display chemotaxis and move into regions of higher activity, if the particles perform work on passive objects, or cargo, to which they are bound. The origin of this cooperative chemotaxis is the exploration of the activity gradient by the active particle when bound to a load, resulting in an average excess force on the load in the direction of higher activity. Using a minimalistic theoretical model, we capture the most relevant features of these active-passive dimers and in particular we predict the crossover between anti-chemotactic and chemotactic behaviour. Moreover we show that merely connecting active particles to chains is sufficient to obtain the crossover from anti-chemotaxis to chemotaxis with increasing chain length. Such an active complex is capable of moving up a gradient of activity such as provided by a gradient of fuel and to accumulate where the fuel concentration is at its maximum. The observed transition is of significance to proto-forms of life enabling them to locate a source of nutrients even in the absence of any supporting sensomotoric apparatus.
\end{abstract}

Escherichia coli is able to steer toward sources of nutrients by altering its tumble rate [1]. While searching, it performs temporal comparisons of nutrient concentrations along its trajectory, and as the concentration increases, the swimmer lowers its tumble rate to move up the concentration gradient of nutrients. This search strategy, known as klinokinesis with adaption, leads to accumulation of bacteria near the top of the nutrient density and thus to chemotaxis [2, 3]. It requires a delicate apparatus for chemical sensing, information processing and a corresponding motoric response, available only to organisms that have reached an advanced level of evolution.

Much simpler are self-propelled particles, which merely adjust their speed in response to the local fuel concentration, a mechanism called orthokinesis. Synthetic Janus particles, an example for this class of swimmers, are driven by catalytic reactions in the presence of e.g. hydrogen peroxide [4] or hydrazine [5]. Biological molecules such as catalytic enzymes have also been reported to show orthokinetic behaviour [6. Instead of intentional tumble movements, induced by active cilia as used by E. coli, these particles change direction randomly through rotational Brownian motion, and, if of roughly spherical shape, the rotational relaxation time is almost independent of the degree of propulsion. Usually, their swim speed (i.e. their degree of activity) is positively correlated with the concentration of fuel. As a consequence, one can show that in presence of a fuel gradient such active Brownian particles (ABPs) accumulate in regions where the fuel concentration is low, known as anti-chemotactic behaviour [7]. This corresponds qualitatively to that of passive Brownian particles in a temperature gradient $[8$, and virtually to all everyday experience that physical bodies accumulate in regions where they are less agitated or moved. This has been recently shown to play a role in the positioning of the nucleus in certain animal cells 9 11]. Although ABPs can display interesting transient behaviour in fuel gradients, called

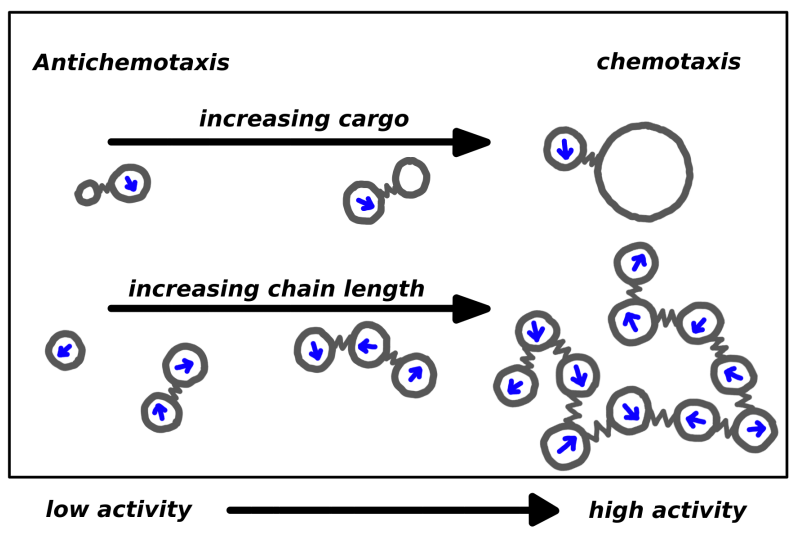

FIG. 1. Crossover from anti-chemotactic to chemotactic behaviour. ABPs are sketched as circles with arrows. Cargo particles (sketched as empty circles) are bound to ABPs. (a) When the cargo is small, that is, its friction coefficient is small, the dimer accumulates in low activity regions (antichemotaxis). When the cargo is large, the dimer accumulates in the high activity regions (chemotactis). (b) Self emergence of cargo in active chains. When active particles are connected in a chain, one observes the crossover from anti-chemotactic to chemotactic behaviour with increasing chain length.

pseudo-chemotaxis [12 15, activity does not lead to an advantage in the search for fuel sources as compared to passive diffusion on long time scales [16].

These observations have led to the general belief that chemotaxis, a prominent feature of living systems, cannot be reflected by uninformed moving objects, in particular not by ABPs. In this work, we demonstrate that ABPs can show chemotactic behaviour in activity gradients when they are bound to a passive cargo particle. With increasing cargo, the active-passive complex switches its behaviour from anti-chemotactic to chemotactic and accumulates in regions with a large fuel concentration (see Fig. 1). Furthermore, we show that an 
explicit distinction between cargo and active particles is not fundamental to our proposed mechanism and can self-emerge in an active system. We demonstrate this in a system of ABPs connected in a chain: with increasing chain length there is a crossover from anti-chemotaxis to chemotaxis.

Our model consists of an ABP [17] attached to a passive cargo by a stiff bond, thus forming a dimer. The activity of the ABP is characterized by its persistence time $\tau$ (characteristic time of rotation of the active particle) and a space-dependent swim force $f_{s}(\boldsymbol{r})$, which we assume to be proportional to the local fuel concentration. Regions where the swim force is large (small) we call high (low) activity regions. The friction coefficient of the ABP is $\gamma$ and that of the cargo is $q \gamma$. The load on the ABP is increased by increasing $q$. Temperature is denoted by $T$ in units such that the Boltzmann constant is unity. In our theoretical approach, we coarse grain the Fokker-Planck equation for the dimer to obtain an effective equation for the 'center-of-friction' coordinate $\boldsymbol{R}$ defined as the friction-weighted vector sum of the position vectors of the $\mathrm{ABP}$ and the load. In this letter, we consider the regime where both the bond length as well as the persistence length of the ABP are small compared to gradients of the swim force. Under these assumptions, the distribution of the ABP is approximately the same as that of the load and therefore $\boldsymbol{R}$ alone can be used to specify the location of the dimer. The steady state distribution of the dimer is obtained by setting the fluxes to zero in the effective equation (see Methods). Our results hold for $d \in\{2,3\}$ dimensions.

Figure 2 displays the stationary density distribution of dimers with a different friction ratio $q$. As long as the cargo is highly mobile ( $\operatorname{small} q$ ), the dimer accumulates in regions of low activity like a single ABP, which - being an orthokinetic swimmer - is anti-chemotactic. When the cargo has large friction (large q), it exhibits chemotaxis and accumulates where the activity is high. The crossover from anti-chemotactic to chemotactic behaviour can be quantitatively captured by our theory (Supplementary Information) which yields the following expression for the steady-state density of the dimer:

$$
\rho(\boldsymbol{R}) \propto\left[1+\frac{1}{1+q} \frac{\tau}{\gamma T d} f_{s}^{2}(\boldsymbol{R})\right]^{-\frac{1}{2} \epsilon}
$$

where

$$
\epsilon=1-q \frac{d-1}{d}
$$

For $q<d /(d-1), \epsilon$ is positive, and the dimer is chemotactic. For $q>d /(d-1), \epsilon$ is negative, and the dimer is anti-chemotactic. At the threshold $q=d /(d-1)$, the distribution of the dimer is uniform and independent of the swim force. Note that in the limit of $q \rightarrow 0$ the density distribution of the dimer reduces to that of a single $\mathrm{ABP}$, and it accumulates in the region of low activity. In general, the exponent $\epsilon$ depends on the potential between

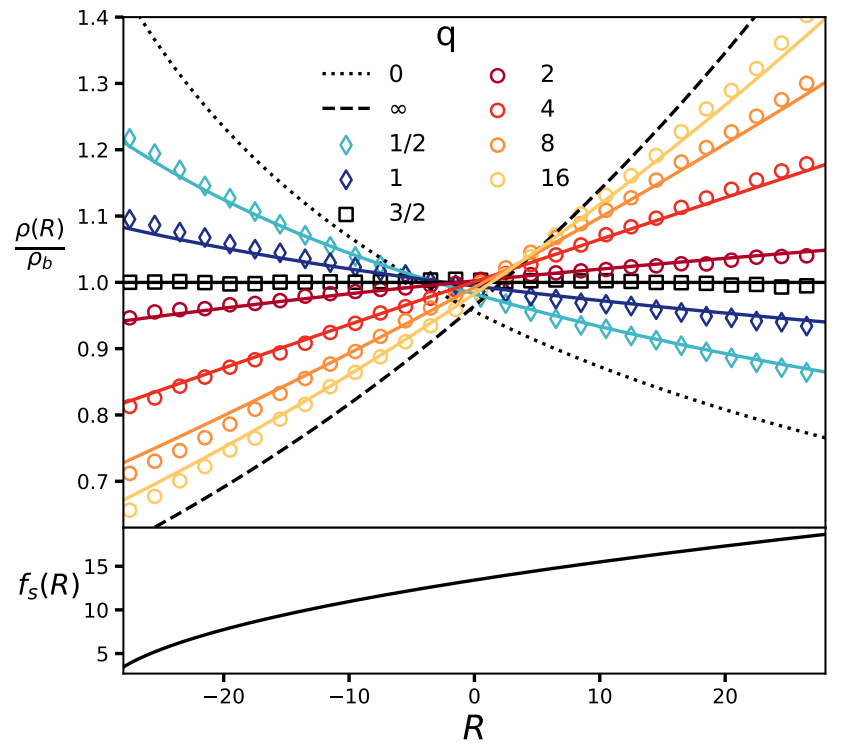

FIG. 2. Density distributions of ABPs in three dimensions rigidly bound to a passive cargo particle with different friction (top figure). Symbols show simulation results; solid lines show the analytical prediction (Eq. (1)). $\rho_{b}$ is the bulk density. The swim force (activity) increases from left to right as $f_{s}(R)=\sqrt{6(R+30)}$ (bottom figure). With a highly mobile cargo particle (blue diamonds) the dimer accumulates in regions of low activity (anti-chemotaxis). At the predicted friction ratio $q=d /(d-1)=3 / 2$ a uniform concentration is found (black squares). With a less mobile cargo (circles) the dimer accumulates where the activity is high (chemotaxis). The dashed line shows the limit of infinite friction of the cargo. This limiting case corresponds to the behaviour of the system under the Born-Oppenheimer approximation in which the cargo particle can be considered as immobile and the ABP moving in a stationary potential. The dotted line shows the limit of vanishing friction of the cargo, where the dimer behaves like a single ABP.

the two particles. In the Supplementary Information we show that the qualitative behaviour of the system is unaffected by the choice of the potential.

In order to understand the mechanism behind the switch from anti-chemotaxis to chemotaxis for increasing $q$, we consider the small and large $q$ limits separately. When the friction coefficient of the cargo particle is much smaller than that of the ABP, the cargo relaxes to its quasi-steady state distribution at each position of the ABP. In this limit, one can consider the dimer to be a single ABP with an increased friction coefficient. Accordingly, the dimer accumulates in the low activity regions (anti-chemotaxis). In the limit of large friction of the cargo particle, the $\mathrm{ABP}$ relaxes to a quasi-steady state distribution at each position of the load particle, and the ABP has enough time to probe the neighbourhood of the cargo particle. The activity gradient results in an effective force on the cargo particle in the direction of the activity gradient. In this limit, one observes the accumulation of the system in high activity regions (chemotaxis). 


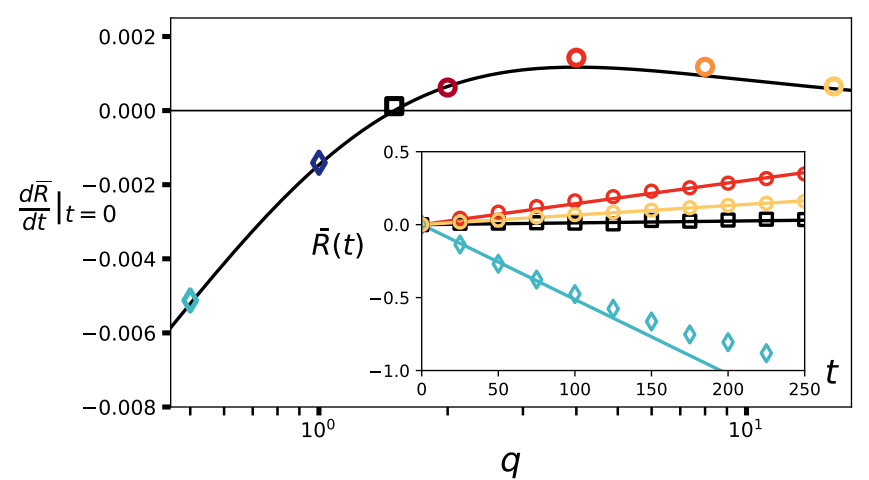

FIG. 3. Time derivative at $t=0$ of the average position of the dimer starting with a uniform distribution $(d \bar{R} / d t)$ with swim force $f_{s}=\sqrt{6(R+30)}$. Symbols show simulation results (colors as in Fig. 22). The line is the theoretical prediction $d \bar{R} / d t \propto \epsilon /(1+q)^{2}$ with the proportionality constant fitted to the data (for details see Supplementary Information). Inset: initial time evolution of the average position $(R(t))$ for several values of $q$. The solid lines show the linear fit for short time from which the data in the main figure is extracted. For $q<3 / 2$ the dimers move to the left down the swim force gradient. For $q>3 / 2$ the dimers move to the right up the swim force gradient. As $q$ increases beyond 4, the dynamics start to slow down due to the increase in the friction of the dimer.

The steady-state density in the large friction limit can be obtained following an independent approach similar to the Born-Oppenheimer approximation in quantum mechanics. When the cargo is large, one can consider it as immobile and the ABP as moving in a stationary potential. The ABP explores the environment around the load and exerts an effective force on it in the direction up the swim force gradient. This effective force can be considered as the driving force for the total system. The steady-state density of a passive Brownian particle with friction $q \gamma$ in a such an effective force field is

$$
\rho(\boldsymbol{R}) \propto \exp \left[\frac{1}{2} \frac{d-1}{d} \frac{\tau}{\gamma T d} f_{s}^{2}(\boldsymbol{R})\right],
$$

which shows that for large $q$ the dimer moves preferentially to regions of high fuel concentration. For details of the calculations, see the SI. Note that this density is the $q \rightarrow \infty$ limit of equation (1). This consideration shows that it is indeed the ability of the active particle to explore the activity gradient that causes the chemotaxis.

Whereas the steady-state distribution measures the chemotactic behaviour of the dimer, it does not shed any light on the 'efficiency' of the chemotactic transport of cargo particles by the ABPs. Though large $q$ results in the strongest chemotactic behaviour, it also slows down the transport of the cargo particle due to the increased friction of the dimer. In our case, this leads to an optimum $q$ that yields the fastest transport to regions of high activity. To quantify this, we use the initial rate of change of the average position of the dimer, starting with

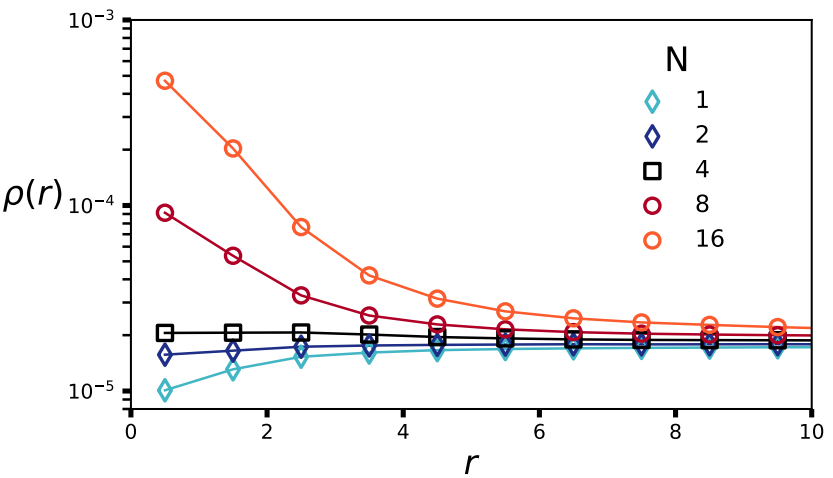

FIG. 4. Stationary concentrations of ABP-molecules inside a spherical container of radius $r_{\max }=25$. The symbols show simulation data, and the lines are a guide for the eye. The source of activity is at its center and the driving force decreases as $f_{s}(r)=15 / r$ for $r \geq 1$, and remains constant $f_{s}(r)=15$ for $r<1$. This activity profile corresponds to a stationary fuel source emitting fuel by diffusion. The monoand dimers are anti-chemotactic and are driven out of the region of high activity (blue diamonds) The quadromer is distributed uniformly (black squares). Longer polymers $(N>4$, red circles) are chemotactic and accumulate near the peak of the fuel concentration.

a uniform distribution (see Fig. 3). At short times, the displacement of the dimer is determined by the convective velocity (Supplementary Information), which is given by $\boldsymbol{V}(\boldsymbol{R}) \propto \nabla f_{s}^{2} \epsilon /(1+q)^{2}$. Depending on the value of $q$, this is either up the swim force gradient (chemotaxis) or down the swim force gradient (anti-chemotaxis). The convective velocity has a maximum at $q=(3 d-1) /(d-1)$ which coincides with the simulation result. Note that for biased movement up the swim-force gradient, only a large enough cargo is necessary, and no memory [18, temporal integration of the fuel concentration [19], or an explicit coupling between the swim-force gradient and the orientation of the ABP [20, 21] is required.

Instead of coupling an ABP to a passive cargo, several ABPs can be combined to form chains or clusters of $N$ 'monomers' 22, 23]. Then, each individual ABP may be regarded as an active pulling agent connected to a 'cargo' of $N-1$ particles because the other $N-1$ ABPs do not pull coherently in identical directions but rather in random directions. Figure 4 displays the stationary radial density distribution profiles of the molecules inside the container. Short oligomers are anti-chemotactic and stay away from regions of high activity. Long polymers, on the other hand, are chemotactic and accumulate where activity is high. The crossover point is seen with the quadromer which is roughly uniformly distributed in the activity field.

Polymeric ABPs are therefore qualitatively similar to a single ABP coupled to a passive cargo: If either the cargo is sufficiently inert or the degree of polymerization sufficiently high, such that it inhibits the free motion of an individual $\mathrm{ABP}$, then the situation of a single $\mathrm{ABP}$ 
inside an approximately stationary potential arises: Inside an activity gradient, the particle is running up the confining potential in the direction of increasing activity. The resulting positional bias generates a net force which - if the system is mobile - drives the same up the activity gradient. If, however, the cargo is rather mobile, then the motion of the ABP remains approximately undisturbed and the complex runs down the activity gradient as an individual ABP does.

ABPs are equivalent to other models of active matter, such as run and tumble [24] active Ornstein-Uhlenbeck particles [25, 26], and the results shown here also apply to these models. We show in the Supplementary Information that the chemotactic behaviour observed in the active-passive dimer cannot be reproduced in a system of dimers in which the active particle is replaced by a passive Brownian particle coupled to a spatially inhomogeneous thermostat.

An interesting outlook is the development of artificial nano-machines which can locate origins of fuel gradients based on the design principle of coupling ABPs to passive cargo without a complex sensing and steering sys- tem. Furthermore, a natural progression of this work is to study how the behaviour of a single active-passive dimer or more complex clusters in an activity gradient affect the collective properties of a system with a large number of such components 2730 .

The coupling of active bodies with or without passive bodies leads to effective sensing of gradients of activity (fuel) and can be generalized in several directions. First, the connectivity between the bodies does not need to be linear as in the studies presented in this letter. Virtually any kind of topology of connecting the active bodies leads to effective chemotaxis, given that the complex provides sufficient friction to single active bodies. Moreover, active bodies moving inside a container that is permeable for the fuel but not for the active bodies can give rise to chemotactic motion of the container, provided the activity gradient is present inside the container. Also in this case, it is the exploration of the active bodies in the gradient field which leads to a higher averaged pressure on the container walls located at the higher activity. Our results may shed light on the origin of prebiotic forms of life and how chemotactic sensing has emerged during evolution.
[1] Howard C. Berg. E. coli in motion. Springer Verlag, Heidelberg, Germany, 2004.

[2] Mark J. Schnitzer, S. M. Block, H. C. Berg, and E. M. Purcell. Strategies for chemotaxis. Symp. Soc. Gen. Microbiol., 46:15, 1990.

[3] Howard C. Berg. Random Walks in Biology. Princeton University Press, Chichester, West Sussex, 1993.

[4] J. R. Howse, R. A. L. Jones, A. J. Ryan, T. Gough, R. Vafabakhsh, and R. Golestanian. Self-Motile Colloidal Particles: From Directed Propulsion to Random Walk. Phys. Rev. Lett., 99(4):048102, July 2007.

[5] Wei Gao, Allen Pei, Renfeng Dong, and Joseph Wang. Catalytic iridium-based janus micromotors powered by ultralow levels of chemical fuels. J. Am. Chem. Soc., 136(6):2276-2279, 2014. PMID: 24475997.

[6] Ah-Young Jee, Sandipan Dutta, Yoon-Kyoung Cho, Tsvi Tlusty, and Steve Granick. Enzyme leaps fuel antichemotaxis. Proceedings of the National Academy of Sciences, 115(1):14-18, 2018.

[7] Mark J. Schnitzer. Theory of continuum random walks and application to chemotaxis. Phys. Rev. E, 48:25532568, Oct 1993.

[8] NG Van Kampen. Diffusion in inhomogeneous media. Zeitschrift für Physik B Condensed Matter, 68(2-3):135138, 1987.

[9] Maria Almonacid, Wylie W Ahmed, Matthias Bussonnier, Philippe Mailly, Timo Betz, Raphaël Voituriez, Nir S Gov, and Marie-Hélène Verlhac. Active diffusion positions the nucleus in mouse oocytes. Nature cell biology, 17(4):470-479, 2015.

[10] Nitzan Razin, Raphael Voituriez, Jens Elgeti, and Nir S Gov. Generalized archimedes' principle in active fluids. Physical Review E, 96(3):032606, 2017.

[11] Nitzan Razin, Raphael Voituriez, Jens Elgeti, and Nir S
Gov. Forces in inhomogeneous open active-particle systems. Physical Review E, 96(5):052409, 2017.

[12] I. R. Lapidus. 'pseudochemotaxis' by micro-organisms in an attractant gradient. Journal of Theoretical Biology, 86:91-103, 1980.

[13] Fei Peng, Yingfeng Tu, Jan C. M. van Hest, and Daniela A. Wilson. Self-guided supramolecular cargoloaded nanomotors with chemotactic behavior towards cells. 127(40):11828-11831, 2015.

[14] Pulak K. Ghosh, Yunyun Li, Fabio Marchesoni, and Franco Nori. Pseudochemotactic drifts of artificial microswimmers. Phys. Rev. E, 92:012114, Jul 2015.

[15] Hidde D. Vuijk, Abhinav Sharma, Debasish Mondal, Jens-Uwe Sommer, and Holger Merlitz. Pseudochemotaxis in inhomogeneous active brownian systems. Phys. Rev. E, 97:042612, 2018.

[16] Holger Merlitz, Hidde D. Vuijk, René Wittmann, Sharma Abhinav, and Jens-Uwe Sommer. Pseudo-chemotaxis of active brownian particles competing for food. Plos One, 15:e0230873, 2020.

[17] Clemens Bechinger, Roberto Di Leonardo, Hartmut Löwen, Charles Reichhardt, Giorgio Volpe, and Giovanni Volpe. Active particles in complex and crowded environments. Rev. Mod. Phys., 88:045006, 2016.

[18] Justus A Kromer, Noelia de la Cruz, and Benjamin M Friedrich. Chemokinetic scattering, trapping, and avoidance of active brownian particles. Physical Review Letters, 124(11):118101, 2020.

[19] M. E. Cates. Diffusive transport without detailed balance in motile bacteria: does microbiology need statistical physics? Rep. Prog. Phys., 75(4):042601, 2012.

[20] Celia Lozano, Borge ten Hagen, Hartmut Löwen, and Clemens Bechinger. Phototaxis of synthetic microswimmers in optical landscapes. Nat. Commun., 7:12828, 
2016.

[21] Celia Lozano and Clemens Bechinger. Diffusing wave paradox of phototactic particles in traveling light pulses. Nature communications, 10(1):1-9, 2019.

[22] Andreas Kaiser, Sonja Babel, Borge ten Hagen, Christian von Ferber, and Hartmut Löwen. How does a flexible chain of active particles swell? The Journal of chemical physics, 142(12):124905, 2015.

[23] Roland G Winkler and Gerhard Gompper. The physics of active polymers and filaments. The Journal of Chemical Physics, 153(4):040901, 2020.

[24] Michael E Cates and Julien Tailleur. When are active brownian particles and run-and-tumble particles equivalent? consequences for motility-induced phase separation. EPL (Europhysics Letters), 101(2):20010, 2013.

[25] Étienne Fodor, Cesare Nardini, Michael E Cates, Julien Tailleur, Paolo Visco, and Frédéric van Wijland. How far from equilibrium is active matter? Physical review letters, 117(3):038103, 2016.

[26] David Martin, Jérémy O'Byrne, Michael E Cates, Étienne Fodor, Cesare Nardini, Julien Tailleur, and Frédéric van Wijland. Statistical mechanics of active ornstein uhlenbeck particles. arXiv preprint arXiv:2008.12972, 2020.

[27] Benno Liebchen and Hartmut Löwen. Synthetic chemotaxis and collective behavior in active matter. Accounts of chemical research, 51(12):2982-2990, 2018.

[28] Suropriya Saha, Ramin Golestanian, and Sriram Ramaswamy. Clusters, asters, and collective oscillations in chemotactic colloids. Physical Review E, 89(6):062316, 2014.

[29] Oliver Pohl and Holger Stark. Dynamic clustering and chemotactic collapse of self-phoretic active particles. Physical review letters, 112(23):238303, 2014.

[30] Babak Nasouri and Ramin Golestanian. Exact phoretic interaction of two chemically active particles. Physical Review Letters, 124(16):168003, 2020.

[31] Hidde Derk Vuijk, Jens-Uwe Sommer, Holger Merlitz, Joseph Michael Brader, and Abhinav Sharma. Lorentz forces induce inhomogeneity and flux in active systems. Physical Review Research, 2(1):013320, 2020.

[32] J.D. Weeks, D. Chandler, and H.C. Anderson. Role of repulsive forces in determining the equilibrium structure of simple liquids. J. Chem. Phys., 54:5237, 1971.

[33] Konstantin V. Klenin, Holger Merlitz, and Jörg Langowski. A brownian dynamics program for the simulation of linear and circular dna and other wormlike chain polyelectrolytes. Biophys. J., 74:780, 1998.

\section{ACKNOWLEDGEMENTS}

A.S. acknowledges support by the Deutsche Forschungsgemeinschaft (DFG) within the project SH 1275/3-1.

\section{AUTHOR CONTRIBUTIONS}

All authors conducted the research together and participated in the preparation and revision of the manuscript.

\section{METHODS}

\section{A. The Dimer Model}

Here we use the standard model of active Brownian particles [17] coupled to a passive Brownian load particle:

$$
\begin{aligned}
\partial_{t} \boldsymbol{r}_{1} & =\frac{1}{\gamma} f_{s}\left(\boldsymbol{r}_{1}\right) \boldsymbol{p}+\frac{1}{\gamma} \boldsymbol{F}+\sqrt{\frac{2 T}{\gamma}} \boldsymbol{\xi}_{1} \\
\partial_{t} \boldsymbol{p} & =\sqrt{2 D_{r}} \boldsymbol{p} \times \boldsymbol{\eta} \\
\partial_{t} \boldsymbol{r}_{2} & =-\frac{1}{q \gamma} \boldsymbol{F}+\sqrt{\frac{2 T}{q \gamma}} \boldsymbol{\xi}_{2}
\end{aligned}
$$

where $\boldsymbol{r}_{1}$ denotes the position of the active particle with friction constant $\gamma$ and swim speed $v\left(\boldsymbol{r}_{1}\right), \boldsymbol{p}$ is a unit vector in the direction of the self-propulsion, and $\boldsymbol{r}_{2}$ is the position of the passive load particle with friction constant $q \gamma$ and $T$ is the temperature in units such that the Boltzmann constant is one. The force binding the two particles together is $\boldsymbol{F}=-\nabla_{\boldsymbol{r}_{1}} U\left(\boldsymbol{r}_{1}-\boldsymbol{r}_{2}\right)$ and $D_{r}$ is the rotational diffusion coefficient. In this letter we consider a stiff potential. The vectors $\boldsymbol{\eta}, \boldsymbol{\xi}$ and $\boldsymbol{\chi}$ are random Gaussian vectors with zeros mean and autocorrelation $\left\langle\boldsymbol{\xi}_{1}^{T}(t) \boldsymbol{\xi}_{1}\left(t^{\prime}\right)\right\rangle=\left\langle\boldsymbol{\xi}_{2}^{T}(t) \boldsymbol{\xi}_{2}\left(t^{\prime}\right)\right\rangle=\left\langle\boldsymbol{\eta}^{T}(t) \boldsymbol{\eta}\left(t^{\prime}\right)\right\rangle=\mathbf{1} \delta\left(t-t^{\prime}\right)$. The rigid bond between the particles is modeled as a harmonic potential,

$$
U\left(\boldsymbol{r}_{1}-\boldsymbol{r}_{2}\right)=\frac{1}{2} k\left(\left|\boldsymbol{r}_{1}-\boldsymbol{r}_{2}\right|-l_{0}\right)^{2},
$$

with equilibrium distance $l_{0}=1$ and spring constant $k$. To make the bond rigid, we take the limit $k \rightarrow \infty$. In this work we set $T=1$, the translational diffusion coefficient $D_{t}=1, D_{r}=20$, and the frictional drag coefficient $\gamma=1$.

In this letter, we focus on the parameter range such that the persistence lenght of the ABP is small compared to gradients in the of the swim force $\left|\nabla f_{s}(\boldsymbol{r})\right| \tau / \gamma \ll 1$, where $\tau=1 /(d-1) D_{r}$ is the autocorrelation time of the orientation vector. Second, we are interested in the parameter range where the separation between the ABP and the load is small compared to gradients of swim force $\left(\boldsymbol{r}_{1}-\boldsymbol{r}_{2}\right) \cdot \nabla_{1} \ln f_{s}\left(\boldsymbol{r}_{1}\right) \ll 1$. In this regime the distribution of the ABP and that of the load are approximately the same because the difference is related to the length scale of the separation.

\section{B. Coarse graining the active-passive dimer}

The Fokker-Planck equation corresponding to Eqs. (4), (5) and (6) is

$$
\begin{gathered}
\partial_{t} P(t)=-\frac{1}{\gamma} \nabla_{1} \cdot\left[\boldsymbol{F P}(t)+f_{s} \boldsymbol{p} P(t)-T \nabla_{1} P(t)\right] \\
+\frac{1}{q \gamma} \nabla_{2} \cdot\left[\boldsymbol{F P} P(t)+T \nabla_{2} P(t)\right]+D_{r} \mathcal{R}^{2} P(t)
\end{gathered}
$$


where $P(t)=P\left(\boldsymbol{r}_{1}, \boldsymbol{p}, \boldsymbol{r}_{2}, t\right)$. We coarse grain this equation in two steps. First we expand in eigenfunctions of the Laplacian on the unit sphere $\boldsymbol{\mathcal { R }}^{2}$ [24, 31], and transform to the 'center-of-friction' coordinate $\boldsymbol{R}=\frac{1}{1+q} \boldsymbol{r}_{1}+\frac{q}{1+q} \boldsymbol{r}_{2}$, which we take as the position of the dimer, and the internal coordinate $\boldsymbol{r}^{\prime}=\boldsymbol{r}_{1}-\boldsymbol{r}_{2}$. Then we integrate out the orientational degrees of freedom $(\boldsymbol{p})$ and the internal coordinate. This results in the following equation for the probability density of the dimer:

$$
\partial_{t} \rho(\boldsymbol{R}, t)=-\nabla \cdot \boldsymbol{J}
$$

where the flux of the dimers is

$$
\boldsymbol{J}=-\frac{1}{2} \epsilon \rho(\boldsymbol{R}, t) \nabla D(\boldsymbol{R})-D(\boldsymbol{R}) \nabla \rho(\boldsymbol{R}, t)
$$

and

$$
D(\boldsymbol{R})=\frac{1}{1+q} \frac{T}{\gamma}+\frac{1}{(1+q)^{2}} \frac{\tau}{d \gamma^{2}} f_{s}^{2}(\boldsymbol{R})
$$

is the course-grained space-dependent diffusion coefficient of the dimer. The zero-flux steady-state solution to Eq. (9) is Eq. (1) in the main text. Details of the calculations can be found in the Supplementary Information.

\section{Brownian Dynamics Simulations}

For the simulations, a coarse-grained model is applied in which all particles are ideal, i.e. no pair interactions except for bond forces are implemented. In confined systems, particles interact with the confining walls via a standard short-range repulsive Weeks-ChandlerAnderson potential 32. Bonds are harmonic potentials as in Eq. (7), with spring constant $k=170$ and equilibrium distance $l_{0}=1$, which sets the unit length of the system. Due to the large spring constant, this is essentially a bond with fixed length.
The time integration of the stochastic differential equations is carried out using a standard second-order Brownian dynamics algorithm 33 . A tentative first-order displacement is

$$
\boldsymbol{r}_{i}^{\prime}(t+d t)-\boldsymbol{r}_{i}(t)=\frac{\boldsymbol{F}_{i}\left(\boldsymbol{r}_{i}, t\right)}{\gamma_{i}} d t+\sqrt{2 D_{t} d t} \boldsymbol{\eta}_{i}(t)
$$

with $i$ the particle index, $d t$ the time step and $D_{t}$ the translational diffusion coefficient. The total force on the $i$ th particle, $\boldsymbol{F}_{i}$, is the sum of all conserved forces (from bonds and bounding walls, if present) and, in case of ABPs, the driving force

$$
\boldsymbol{f}_{i}\left(\boldsymbol{r}_{i}, t\right)=f_{s}\left(\boldsymbol{r}_{i}\right) \boldsymbol{p}_{i}(t)
$$

with the coordinates $\boldsymbol{r}_{i}$, the coordinate-dependent activity $f_{s}\left(\boldsymbol{r}_{i}\right)$, and the particle-embedded unit orientation vectors $\boldsymbol{p}_{i}(t)$. The stochastic vectors $\boldsymbol{\eta}_{i}(t)$ are Gaussian distributed with zero mean and time correlations $\left\langle\boldsymbol{\eta}_{i}(t) \boldsymbol{\eta}_{j}^{T}\left(t^{\prime}\right)\right\rangle=1 \delta\left(t-t^{\prime}\right) \delta_{i j}$. The second half-step is

$$
\boldsymbol{r}_{i}(t+d t)-\boldsymbol{r}_{i}^{\prime}(t+d t)=\frac{-\boldsymbol{F}_{i}\left(\boldsymbol{r}_{i}, t\right)+\boldsymbol{F}_{i}^{\prime}\left(\boldsymbol{r}_{i}^{\prime}, t+d t\right)}{2 \gamma_{i}} d t
$$

where $\boldsymbol{F}_{i}^{\prime}\left(\boldsymbol{r}_{i}^{\prime}, t+d t\right)$ are the forces calculated for the advanced conformation $\boldsymbol{r}_{i}^{\prime}(t+d t)$. Finally, the orientation vectors of the ABPs are updated via

$$
\boldsymbol{p}_{i}(t+d t)-\boldsymbol{p}_{i}(t)=\sqrt{2 D_{r} d t} \boldsymbol{\eta}_{i}(t) \times \boldsymbol{p}_{i}(t)
$$

For all simulations in this work, we used a timestep of $d t=10^{-3}$.

Because the theory does not incorporate boundary effects, we also ignore these effects in Fig. 2. We do this by removing the data closer than $\Delta$ to either of the walls and rescaling the density such that $\int_{\Delta-L / 2}^{-\Delta+L / 2} d R \rho(R)=1$, with $\Delta=2$. 